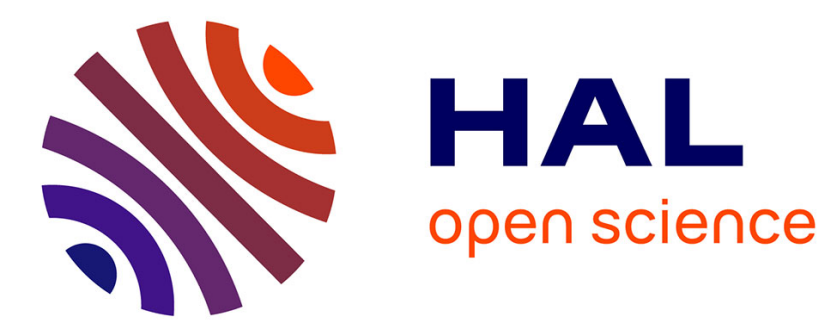

\title{
Étude des conditions de démarrage d'un propulseur sous-marin à vis d'Archimède en appui sur une argile
}

\author{
R. Barriol, A. Goutierre, J.P. Longuemard, A. Valls
}

\section{To cite this version:}

R. Barriol, A. Goutierre, J.P. Longuemard, A. Valls. Étude des conditions de démarrage d'un propulseur sous-marin à vis d'Archimède en appui sur une argile. Revue de Physique Appliquée, 1985, 20 (12), pp.837-844. 10.1051/rphysap:019850020012083700 . jpa-00245400

\section{HAL Id: jpa-00245400 https://hal.science/jpa-00245400}

Submitted on 1 Jan 1985

HAL is a multi-disciplinary open access archive for the deposit and dissemination of scientific research documents, whether they are published or not. The documents may come from teaching and research institutions in France or abroad, or from public or private research centers.
L'archive ouverte pluridisciplinaire HAL, est destinée au dépôt et à la diffusion de documents scientifiques de niveau recherche, publiés ou non, émanant des établissements d'enseignement et de recherche français ou étrangers, des laboratoires publics ou privés. 


\title{
Étude des conditions de démarrage d'un propulseur sous-marin à vis d'Archimède en appui sur une argile
}

\author{
R. Barriol $\left({ }^{+}\right)$, A. Goutierre $\left({ }^{+}\right)$, J. P. Longuemard $\left({ }^{*}\right)$ et A. Valls $\left({ }^{+}\right)$ \\ $\left(^{+}\right)$Université de Perpignan, Laboratoire de Mécanique, d'Acoustique et d'Instrumentation, \\ Avenue de Villeneuve, 66025 Perpignan Cedex, France \\ (*) Ecole Centrale des Arts et Manufactures, Grande Voie des Vignes, 92290 Châtenay-Malabry, France
}

(Reçu le 23 novembre 1984, révisé le 16 juillet 1985, accepté le 2 août 1985)

\begin{abstract}
Résumé. - Cette étude décrit le comportement, en régime transitoire, de la vitesse angulaire d'une vis d'Archimède entraînée par un moteur à courant continu et prévue pour la portance et la propulsion d'un vecteur sous-marin destiné à des travaux par grands fonds. Cette dernière est en appui sur une argile cohésive et à forte teneur en eau. La mise en équation du phénomène est faite à partir de la connaissance du coefficient de frottement argile métal en fonction de la vitesse de cisaillement (reliée à la vitesse angulaire à travers le rayon et le pas de vis). Une solution numérique est présentée ainsi qu'une formule approchée qui permet, à partir de deux facteurs (une constante de temps et un exposant), de comparer les résultats expérimentaux obtenus en caisson hyperbar aux résultats théoriques. L'étude du courant électrique est abordée suivant deux critères : 1) le courant de démarrage (couple maximal), 2) le régime permanent en vue de l'autonomie.
\end{abstract}

\begin{abstract}
This article describes the transitory functioning of an Archimedes' screw for supporting and propelling a submarine vehicle-powered by a d.c. motor on a sea bed made up of cohesive clay with a high water content. An equation is given to describe the behaviour of the screw, using the coefficient of friction between metal and clay in ratio to the cutting speed (linked to the angular speed by the radius and pitch of the screw). An numerical solution is exposed and a formula using two factors (a time constant and a exponent) enables the experimental data is Caisson hyperbars to be compared to the experimental solution with the basic equation. The variation in the intensity of the electric current in ratio to time is also analysed and described for two case : 1) in start working, 2) in normal working. This is necessary for the determination of the motor.
\end{abstract}

\section{Notations :}

$\begin{array}{ll}a, b, c & \begin{array}{l}: \text { coefficients définissant le frottement } \\ \text { en fonction de la vitesse. }\end{array} \\ f & : \text { coefficient de frottement. } \\ n & : \text { force contre électromotrice fonction } \\ & \text { de la vitesse angulaire. } \\ t & : \text { temps. } \\ v & : \text { vitesse linéaire. }\end{array}$

$A, B, C, D, E, F^{\prime}:$ (constante utilisée pour modéliser la fonction).

$E^{\prime} \quad$ : force contre électromotrice.

F $\quad$ :force longitudinale appliquée par la vis.

I $\quad$ : courant électrique.

$J \quad$ : moment d'inertie.

K : constante de couple du moteur.

$L \quad$ : inductance.

$R \quad$ : rayon de la vis.
: surface de contact argile-métal. : résistance électrique du moteur. : constante de temps. : couple résistant. : vitesse angulaire.

\section{Introduction.}

L'exploitation des richesses sous-marines implique de plus en plus souvent l'exécution de travaux à partir de robots. Les plus connus d'entre eux (ramassage des nodules dans le futur, la pose et le contrôle du tube conducteur en appui sur le fond, l'implantation de système à poste fixe) impliquent l'existence de vecteurs présentant une grande capacité de manœuvre ou de réaction $[5,8,11]$.

Certains de ces travaux sont effectués à vitesses lentes et nécessitent une grande force de propulsion 
pour l'avance du véhicule. Une hélice en rotation dans l'eau permet d'obtenir des vitesses appréciables mais n'a pas de couple suffisant à bas régimes, et les charges à ramener ou à déplacer peuvent être fort importantes, d'où l'idée de réaliser un engin en appui sur le fond [4-10] supporté par des chenilles ou des vis d'archimèdes [1-3, $5,12,13]$. Le choix de l'un de ces systèmes découle des qualités mécaniques du fond sur lequel l'appareil concerné œuvre [4]. On doit donc considérer la situation géographique où les travaux sont programmés. En effet, les roues et les chenilles qui conviennent pour les fonds sableux, généralement côtiers ou situés sur les plateaux continentaux, ne semblent pas convenir pour les fonds argileux à forte teneur en eau (donc fortement compressibles) présents dans les grands fonds, dans les milieux estuariens ou encore dans les lagunes margino-littorales [11]. Un système à vis d'archimède contrarotatives qui compensent les efforts transversaux présente l'avantage d'une grande surface de portance, comparable à celle d'une chenille, et de provoquer un sillon (portance supplémentaire égale au poids de l'argile déplacée) assurant une certaine sécurité. Le sillon permet également une stabilité latérale (talus ou pente...). Les filets, en appui horizontal, peuvent présenter une grande section d'appui, d'où la possibilité d'avoir un effort longitudinal important $[7,10]$. Nous nous proposons, dans le cadre de cette étude, d'analyser le comportement de la vitesse de rotation (du moteurréducteur) et du courant électrique.

\section{Contexte de l'étude.}

Les paramètres nécessaires à la prévision du comportement d'un groupe motopropulseur de ce type sont classés en deux groupes qui concernent :

- la modélisation du comportement de la vis d'archimède,

- l'analyse de la chaîne vis-transmission-moteur.

La première, étudiée par ailleurs $[4,7,10]$ se décompose elle-même en plusieurs parties :

- portance,

- propulsion,

- effort limite transmissible (limité par la réaction horizontale du sol),

- effort au démarrage et en régime permanent (couple).

Le deuxième aspect concerne la connaissance du courant au démarrage et en palier nécessaire pour définir certaines caractéristiques de la source d'énergie (courant de pointe, énergie à emmagasiner ou masse de batterie d'accumulateurs) à prévoir ainsi que pour le dimentionnement du moteur.

Le couple résistant découle de deux états :

- le fonctionnement normal dès que la vis a commencé à tourner,

- le couple de cisaillement provoqué par l'adhérence du sol au métal qui est indépendant des efforts à transmettre et qui n'existe qu'avant la rotation de la vis. Ce type de comportement étudié par ailleurs exige la prévision d'un courant électrique de pointe suffisant pour rompre ce contact. La sécurité de fonctionnement nécessite également cette connaissance.

La composition des forces exercées par la vis en appui sur des argiles découle du frottement fonction de la vitesse tangentielle, de l'effort à transmettre, de la force d'appui sur le sol, de l'effort nécessaire pour tracer le sillon. Elle se simplifie en ramenant le tout à une résultante axiale en appuis horizontal sur les filets des vis. Le couple résistant est alors descriptible par :

$$
\Gamma_{\mathrm{r}}=\varphi(R, F, f)
$$

où

$R$ est le rayon,

$F$ est la force longitudinale résultante appliquée par la vis,

$f$ est le coefficient de frottement métal-sédiment.

L'effort réel d'appui provoqué par les filets dépend de l'angle de pas de vis qui, pour un rendement optimal, est fonction de la vitesse tangentielle des éléments en contact avec le sol [10]. La réaction argile métal est également fonction du temps de contact $\left(t_{\mathrm{c}}\right)$. L'adhérence, qui pour une faible valeur de $t_{\mathrm{c}}$ est proche du tiers de la cohésion $(c)$, peut atteindre une valeur égale à $c$ lorsque $t_{\mathrm{c}}$ est voisin de 24 heures. Le problème posé sera alors d'obtenir un couple instantané tel que :

$$
\Gamma_{\mathrm{r}}=\sum R S_{n} \tau_{n}
$$

où :

$R$ est le rayon de vis,

$S_{n}$ sont des éléments de surface de contact,

$\tau_{n}$ est l'adhérence métal argile de l'élément de surface $n$.

Cette dernière relation permet le calcul du courant maximal à prévoir au cas où $\tau_{n}$ soit égale à $c$ et concerne donc l'aspect sécurité de démarrage.

En régime d'avance normal le comportement découle d'un frottement $f$ qui, en fonction de la vitesse longitudinale $(v)$, a une valeur numérique définie par $[3,10,13]$ :

$$
f=a+b v+c v^{2} \text {. }
$$

La constante (a) pourrait éventuellement représenter de l'adhérence argile-métal, il y aurait alors lieu de tenir compte du temps d'arrêt de l'engin et la considérer comme pouvant avoir deux états. Compte tenu de l'objectif il nous a semblé plus simple de traiter séparément les deux cas.

La relation (3) établie à partir de la connaissance de la force de traction de skis en appui sur une argile permet de transposer certains résultats acquis en nature $[4,13]$ à la prévision de comportement de vis soit :

$$
\Gamma_{\mathrm{r}}=R \cdot F \cdot\left[a+b v+c v^{2}\right],
$$


sachant que $v=R \omega$, il vient :

$$
\Gamma_{\mathrm{r}}=R \cdot F \cdot\left[a+b R \cdot \omega+c(R \omega)^{2}\right] .
$$

Le type de moteur électrique retenu pour délivrer l'énergie mécanique est à courant continu à aimant permanent sans collecteur, ce qui permet de mettre un fluide isolant maintenant l'ensemble en équipression (500 bars). Il n'y aura donc pas de déformation mécanique due au travail en haute pression hydrostatique risquant de bloquer la rotation de l'ensemble. Le stator est directement relié aux batteries d'alimentation. Ce type de moteur ne supporte pas de surintensité, même durant un court instant, sans risque de destructions de l'aimentation, d'où l'intérêt d'une bonne modélisation du démarrage.

Les paramètres représentatifs du comportement du moteur utilisé pour les expériences, sont :

- la résistance de l'enroulement $\rho$,

- l'inductance $L$,

- le facteur de couple $K(\Gamma=K I)$,

- le facteur reliant la force électromotrice $E^{\prime}$ à la vitesse angulaire $\omega\left(E^{\prime}=\omega n\right)$,

- le moment d'inertie $J_{\text {mot }}$.

\section{Etude théorique.}

Deux cas types de démarrage sont à analyser, un au cours duquel $\omega$ croît, un qui correspond au décollage $\mathrm{du}$ cylindre. Ce phénomène a lieu lorsqu'il y a eu un temps de contact avant rotation de plusieurs heures, voire de plusieurs jours. Il faut obtenir un couple suffisant pour rompre le contact.

Nous étudierons la variation de $\omega(t)$ pour le cas le plus courant (comportement de la vis suivant une loi de frottement) puis nous étendrons la modélisation en fonction d'une éventuelle adhérence.

3.1 ETUDE DE LA VITESSE DE ROTATION AVEC ADHÉRENCE NULLE OU APRÈS RUPTURE D'ADHÉRENCE. - La vitesse de rotation de la vis et la tension d'alimentation du moteur délivrée par des batteries en équipression conditionne la valeur du courant dans le moteur :

$$
U-n \omega=\rho I+L \frac{\mathrm{d} I}{\mathrm{~d} t} .
$$

Le courant provoque un couple destiné à vaincre l'inertie du système (provoqué par le moment du moteur $J_{\text {mot }}$ et celui de la vis $J_{\text {vis }}$ ) et la résistance due au frottement.

Nous considérerons donc que le couple ramené aux filets a pour expressions :

$$
\Gamma_{\mathrm{r}}=J_{\text {rés }} \frac{\mathrm{d} \omega}{\mathrm{d} t}+R \cdot F \cdot\left[a+b R \omega+c(R \omega)^{2}\right]
$$

où :

$$
J_{\text {mot }}+J_{\text {vis }}=J_{\text {rés' }^{\prime}} .
$$

Le courant est proportionnel au couple donc :

$$
\frac{\partial I}{\partial t}=\frac{1}{K} \frac{\partial \Gamma}{\partial t}
$$

d'où :

$$
\begin{aligned}
\frac{\partial I}{\partial t}=\frac{1}{K}\left[J_{\text {rés }} \frac{\omega}{t^{2}}\right. & +R \cdot F . \times \\
& \left.\times\left(-1,18 R \frac{\partial \omega}{\partial t}+2 R^{2} w \frac{\partial \omega}{\partial t}\right)\right] .
\end{aligned}
$$

En reportant (7) et (9) dans (5) l'équation représentant le comportement devient :

$$
\begin{aligned}
& U-n \omega=\frac{\rho}{K}\left(\left[J_{\text {rés }} \frac{\partial \omega}{\partial t}+R \cdot F \cdot\left(a+b R \omega+c w^{2} R^{2}\right)\right]+\right. \\
& \left.+\frac{L}{K}\left[J_{\text {rés }} \frac{\partial^{2} \omega}{\partial t^{2}}+R \cdot F \cdot\left(b R \frac{\partial \omega}{\partial t}+2 c R^{2} \omega \frac{\partial \omega}{\partial t}\right)\right]\right) .
\end{aligned}
$$

Fig. 1. - Schéma d'une vis d'Archimède.

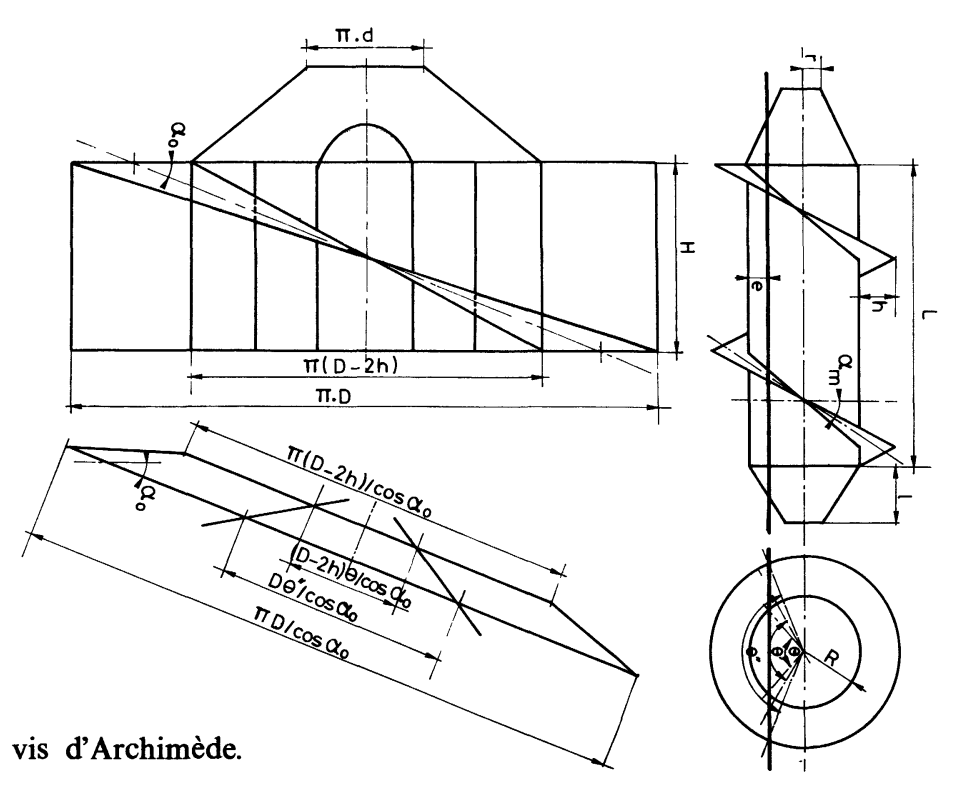

[Scheme of a Archimede screw.] 
Si l'on pose :

$$
\begin{gathered}
K U-a F R . \rho=A, \\
J_{\text {rés }} L=B, \\
\rho J_{\text {rés }}+b R^{2} F L=C, \\
K_{n}+B R^{2} F=D, \\
2 c L R^{3} F=E \text { et } c p R^{3}=F^{\prime},
\end{gathered}
$$

l'équation (10) se résume à :

$$
A=B \frac{\partial^{2} \omega}{\partial t^{2}}+C \frac{\partial \omega}{\partial t}+D \omega+E \omega \frac{\partial \omega}{\partial t}+F^{\prime} \omega^{2} .
$$

La solution numérique de cette relation sera présentée au paragraphe 4 (analyse expérimentale en fonction de la valeur des facteurs $A, B, C, D, E$ et $F$ ).

\subsection{ETUde DU COURANT.}

\subsubsection{En fonction de la loi de frottement.}

$$
I=\frac{1}{K}\left[J_{\text {rés }} \frac{\partial \omega}{\partial t}+R \cdot F .\left(a+b R \omega+c R^{2} \omega^{2}\right)\right] .
$$

En posant :

$$
J / K=G ; \frac{R F}{K} \cdot a=H ; b \frac{R^{2} F}{K}=M ; \frac{R^{3} F c}{K}=N,
$$

il est possible de relier $I$ à $\omega$ par :

$$
I=G \cdot \frac{\partial \omega}{\partial t}+M \omega+N \omega^{2}+H
$$

3.2.2 Cas particulier de l'adhérence. - La vitesse de rotation est nulle, l'équation générale du comportement du courant est :

$$
\begin{aligned}
& I=\frac{U}{\rho}\left(1-\exp -\frac{t}{\tau_{\mathrm{m}}}\right), \\
& \text { où } \tau_{\mathrm{m}}=L / \rho .
\end{aligned}
$$$$
\text { Le couple produit par } I \text { vaut : }
$$

$$
\Gamma=K \quad \frac{U}{\rho}\left(1-\exp -\frac{t}{\tau_{\mathrm{m}}}\right),
$$

il doit atteindre une valeur égale à

$$
\Gamma_{\mathrm{r}}=R S_{n} \tau_{n^{\prime}}
$$

pour provoquer un début de rotation. Dès lors le comportement réagit suivant les relations (7) et (11) :

$$
I=\frac{\sum R S_{n} \tau_{n}}{K}
$$

La solution numérique, pour ces deux configurations extrêmes, conduit aux figures $4 a$ et $b$.

Le courant croît rapidement puis décroît dès que le cylindre tourne pour tendre vers sa valeur nominale.
Toutefois le courant de pointe est beaucoup plus élevé dans le cas d'une forte adhérence ( $a$ garde une valeur proche de 1 pendant un court instant).

\section{Analyse expérimentale. Comparaison avec la théorie (fonction représentation).}

4.1 Conditions D'ANALYSE. - Les conditions d'analyse en haute pression nécessite l'implantation d'un ensemble de mesures dans un caisson hyperbar comprenant (Fig. 2) :

- un bac à sédiment,

- le système de contrôle du sédiment,

- un moteur entraînant un cylindre en appui sur le sédiment pour lequel $F$ est identique à celui d'un filet en appui sur de l'argile ( $F$ est égale à $\mathrm{mg}$ ).

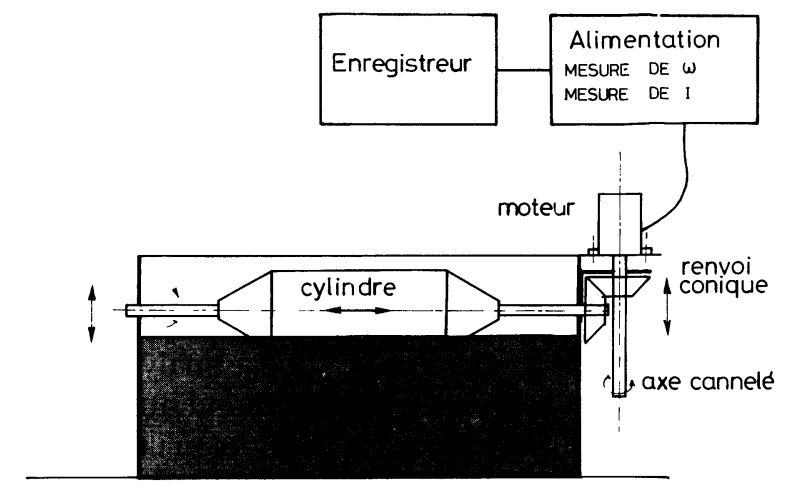

Fig. 2. - Schéma de l'expérience.

[Synoptic of experimental device.]

L'ensemble de mesure prévu pour fonctionner sous une pression de $6 \times 10^{7} \mathrm{~Pa}$ présente un volume relativement réduit (de l'ordre de $1,5 \mathrm{~m}^{3}$ ) mais simule correctement les conditions ambiantes régnant par grands fonds. Les dimensions du cylindre, donc du moteur, sont identiques à celles d'un véhicule expérimental testé en mer en site côtier. Les sédiments utilisés reconstitués à partir de bentonite représentant les caractéristiques des argiles des fonds à nodules (cohésion comprise entre 500 et $3000 \mathrm{~Pa}$, masse volumique de $1400 \mathrm{~kg} \mathrm{~m}^{-3}$ ).

Le volume du caisson utilisé conduit à des valeurs de $F$ de l'ordre de $25 \mathrm{~N}$ compatibles avec un moteur dont la tension d'alimentation est de $28 \mathrm{~V}$ pour un courant maximal de $5 \mathrm{~A}$ assurant un couple de $4,2 \mathrm{mN}$. Le moment d'inertie global (cylindre + moteur) est de $9,7 \times 10^{-3} \mathrm{~kg} \cdot \mathrm{m}^{-2}$ pour un rayon $R$ de $0,0625 \mathrm{~m}$.

Sachant que $K=0,848 \mathrm{mN}$. A ${ }^{-1}$ et que $n=$ $0,848 \mathrm{~V} \mathrm{rad}^{-1} \cdot \mathrm{s}^{-1}$, il vient (pour $a=0,4 ; b=-1,18$; $c=1 ;[4,10,11])$ :

$$
\begin{array}{lll}
A=20,05 & B=12,9 \times 10^{-5} & C=5,2 \times 10^{-2} \\
D=8 \times 10^{-2} & E=1,82 \times 10^{-4} & F^{\prime}=3 \times 10^{-2} .
\end{array}
$$


4.2 Solution DE L'ÉQuation (11). Relation DE COMPARAISON. - L'équation (11) a alors la valeur suivante :

$20,05=1,29 \times 10^{-4} \frac{\partial^{2} \omega}{\partial t^{2}}+5,2 \times 10^{-2} \frac{\partial \omega}{\partial t}+$

$+8 \times 10^{-2} \omega+1,82 \times 10^{-4} \omega \frac{\partial \omega}{\partial t} \times 4 \times 10^{-2} \omega^{2}$

Ce type d'équation peut admettre une solution plus une solution plus particulière mais la valeur même des coefficients interdit l'existence d'oscillations, il semble préférable :

- de proposer une solution numérique obtenue par la méthode des différentes finies (Fig. 3). Le cylindre a un temps de démarrage relativement long, sa vitesse de rotation tend par la suite vers un état d'équilibre de manière quasi exponentielle ;

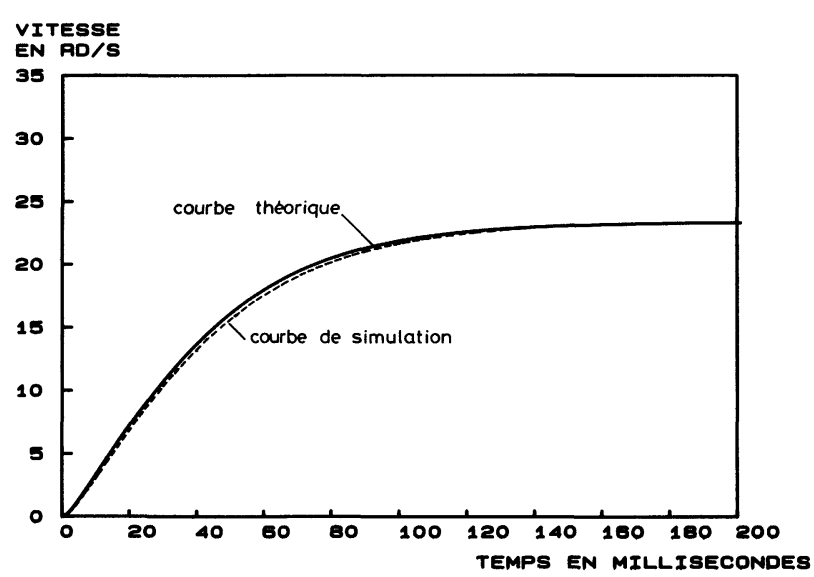

Fig. 3. - a) Solution numérique de l'équation (13). b) Solution obtenue par équation de simulation.

[a) Numerical solution of equation (13). b) Plotting with an semi-empirical law.]

- d'établir un moyen de comparaison entre les résultats expérimentaux représentés sous forme de courbes et la modélisation numérique effectuée à partir de l'équation (11). La recherche d'une loi expérimentale de comportement représentative du comportement théorique suppose une connaissance du phénomène à étudier et la quantification d'un nombre de paramètres aussi restreint que possible. Une fonction de simulation de forme exponentielle rend parfaitement compte de la vitesse en fonction du temps :

où :

$$
\omega=\omega_{0}\left[1-\exp -\left(\frac{t}{\tau}\right)^{n}\right]
$$

- $\omega_{0}$ est calculée pour des valeurs nulles des dérivées première et seconde de l'équation (11), d'où :

$$
20,5=8 \times 10^{-2} \omega_{0}+4 \times 10^{-2} \omega_{0}^{2}
$$

$\omega_{0}$ ne pouvant être nulle ou négatif, la solution de l'équation est : $\omega_{0}=23,24 \mathrm{rad} . \mathrm{s}^{-1}$, ce qui correspond à une vitesse tangentielle de $1,4 \mathrm{~m} \cdot \mathrm{s}^{-1}$ qui, compte tenu du pas de vis est parfaitement représentative de ce qui pourrait se passer pour un engin d'exploitation sousmarine.

- les valeurs respectives de $\tau$ et de $n$ sont déductibles de valeurs de $\omega^{\prime}$ correspondant à des temps $(t)$.

De l'équation (17), nous obtenons :

$$
\frac{\partial \omega}{\partial t}=\omega^{\prime}=\omega_{0} \cdot n\left(\frac{t}{\tau}\right)^{n-1} \cdot \exp -\left(\frac{t}{\tau}\right)^{n} .
$$

De deux valeurs de $\omega^{\prime}$ correspondant aux temps $t_{1}$ et $t_{2}$, il vient :

$$
\begin{aligned}
\log \frac{\omega^{\prime}\left(t_{1}\right)}{\omega^{\prime}\left(t_{2}\right)}=(n & -1) \log \left(\frac{t_{1}}{t_{2}}\right)+ \\
& +\left[\left(\frac{t_{2}}{\tau}\right)^{n}-\left(\frac{t_{1}}{\tau}\right)^{n}\right] .
\end{aligned}
$$

Par une opération similaire avec $3^{\mathrm{e}}$ temps $t_{3}$ et en posant $t_{1}=x t_{2}$ et $t_{2}=x t_{3}$, on peut écrire en soustrayant l'équation $\omega^{\prime}\left(t_{2}, t_{3}\right)$ de $\omega^{\prime}\left(t_{1}, t_{2}\right)$ :

$$
\log \frac{\omega^{\prime}\left(t_{1}\right) \omega^{\prime}\left(t_{3}\right)}{\left(\omega^{\prime}\left(t_{2}\right)\right)^{2}}=\left(\frac{t_{3}}{\tau}\right)^{n}\left(2 x^{n}-x^{2 n}-1\right) .
$$

Une deuxième opération semblable conduite avec des temps $t_{2}, t_{3}$ et $t_{4}$ tels que $t_{2}=x t_{3}=x t_{4}$ conduit à une équation analogue :

$$
\log \frac{\omega^{\prime}\left(t_{2}\right) \cdot \omega^{\prime}\left(t_{4}\right)}{\left(\omega^{\prime}\left(t_{3}\right)\right)^{2}}=\left(\frac{t_{4}}{\tau}\right)^{n}\left(2 x^{n}-x^{2 n}-1\right) .
$$

Il est alors possible d'éliminer $\tau$ et de calculer $n$,

$$
\begin{aligned}
& \log \frac{\omega^{\prime}\left(t_{1}\right) \cdot \omega^{\prime}\left(t_{3}\right)}{\left(\omega^{\prime}\left(t_{2}\right)\right)^{2}} / \log \frac{\omega^{\prime}\left(t_{2}\right) \cdot \omega^{\prime}\left(t_{4}\right)}{\left(\omega^{\prime}\left(t_{3}\right)\right)^{2}}= \\
&=\left(\frac{t_{3}}{\tau}\right) /\left(\frac{t_{4}}{\tau}\right)^{n}=\left(\frac{t_{3}}{t_{4}}\right)^{n}
\end{aligned}
$$

$\left(t_{3} / t_{4}\right)^{n}$ est égal au produit de deux constantes correspondant à deux couples de valeurs triples de $\omega^{\prime}(t)$.

$$
\omega^{\prime}\left(t_{1}\right), \omega^{\prime}\left(t_{2}\right), \omega^{\prime}\left(t_{3}\right) \text { et } \omega^{\prime}\left(t_{2}\right), \omega^{\prime}\left(t_{3}\right), \omega^{\prime}\left(t_{4}\right) \text {. }
$$

Connaissant $n$ il est facile de déduire $\tau$. Ces deux facteurs valent respectivement 1,23 et 0,045 . La courbe de simulation obtenue à partir de la relation (19) présente un coefficient de corrélation avec celle obtenue à partir de l'équation (11) de 0,98 . On peut donc comparer les données expérimentales obtenues à partir de $n$ et $\tau$ avec la formulation exacte à travers l'expression (19) et utiliser ces deux paramètres lors du calcul des vis et des motoréducteurs les entraînant.

4.3 ANALySE DES DONNÉES EXPÉRIMENTALES. - Les séries d'essais conduits en caisson hyparbar ont permis 


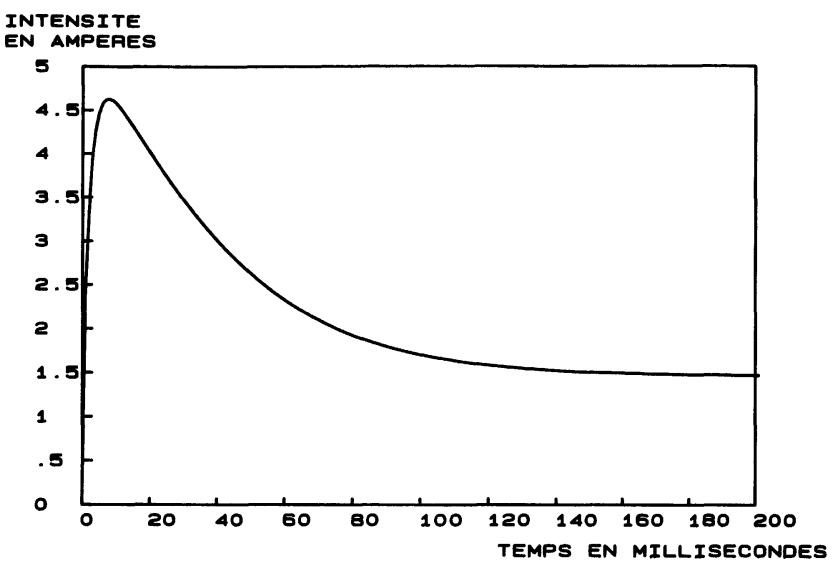

a)

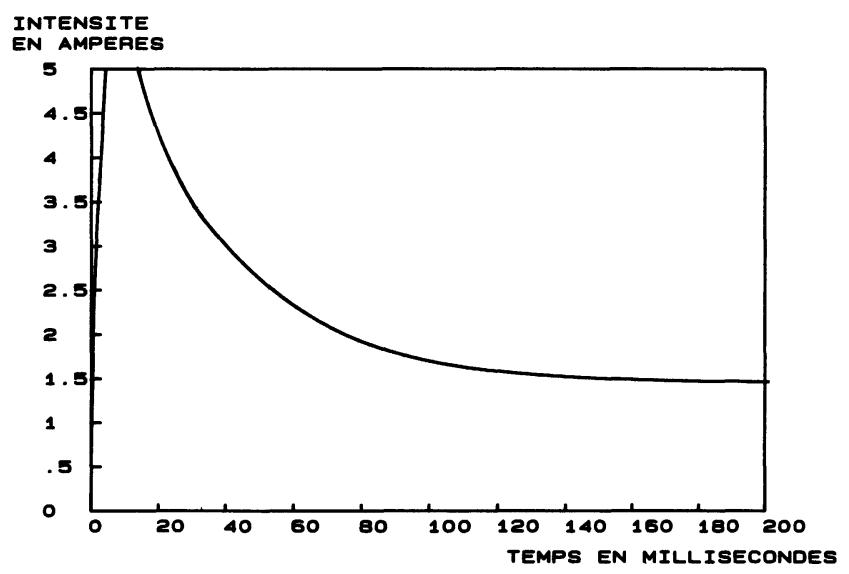

b)

Fig. 4. - Evolution du courant en fonction du temps (solution numérique). a) Sans adhérence loi de frottement. b) Avec adhérence.

[Electrical current (time law). a) Without friction. b) With friction.]

d'obtenir des courbes traduisant l'évolution de $\omega(t)$ et de $I(t)$ (Figs 3 et 4 ).

4.3.1 Vitesse angulaire. - Les points de comparaison entre courbes théoriques et courbes expérimentales (Tabl. I) sont fournis par $\tau$ et par $n$. Ils concernent des argiles artificielles réalisées à partir de bentonite qui, mélangée à $75 \%$ d'eau, reproduisent certaines caractéristiques géomécaniques des argiles des grands fonds (cohésion donc potance et teneur en eau). Le temps de constitution du sédiment (de 15 jours à 3 semaines) implique une limitation du nombre d'expériences. Nous ne disposons que de quatre séries d'essais résumés ci-dessous.

L'expérience confirme le fait qu'il n'y a pas d'oscillations amorties en régime transitoire, ce qui est essentiellement dû au comportement viscoélastique de l'argile.

Le comportement de l'ensemble moto-propulseur est suffisament décrit par la relation (19) et l'on peut prévoir le courant du moteur.
Tableau I. - Résumé de valeurs expérimentales concernant la vitesse angulaire $\left(\omega_{0}\right)$. Expériences $N^{\circ}$

\begin{tabular}{lllll}
\hline $\begin{array}{c}\text { Valeurs mesurées } \\
\text { Valeurs théoriques }\end{array}$ & 1 & 2 & 3 & 4 \\
\hline$\tau 0,045$ & 0,05 & 0,06 & 0,05 & 0,06 \\
\hline$n 1,23$ & 1,23 & 1,23 & 1,23 & 1,23 \\
\hline
\end{tabular}

Le temps nécessaire pour atteindre le régime permanent est plus long que prévu par la théorie. Le fait que l'argile reste attachée au cylindre même en rotation peut expliquer en partie ce fait par augmentation du moment d'inertie de l'ensemble et par augmentation du coefficient de frottement (le cisaillement argileargile a un effet plus important que le frottement argile-métal).

4.3.2 Alimentation en énergie. - L'équation (14) qui définit le courant en fonction de $f(v)$ peut également être résolu numériquement (Fig. 4). Le comportement global de ce dernier, qui découle des caractéristiques électriques du moteur et du couple résistant, croît vite pour atteindre sa valeur nominale (Fig. 4). Toutefois, il est nécessaire que ce dernier ait une valeur égale à celle minimum provoquant un début de rotation du moteur pour obtenir $\omega(t)$. Nous n'avons pu, pour des raisons pratiques, mesurer le comportement au démarrage dans le cas d'une adhérence égale à la cohésion du sédiment.

La valeur de crête du courant peut être quatre à cinq fois supérieure à l'intensité en régime permanent. Ce cas particulier rare correspond à celui d'un engin
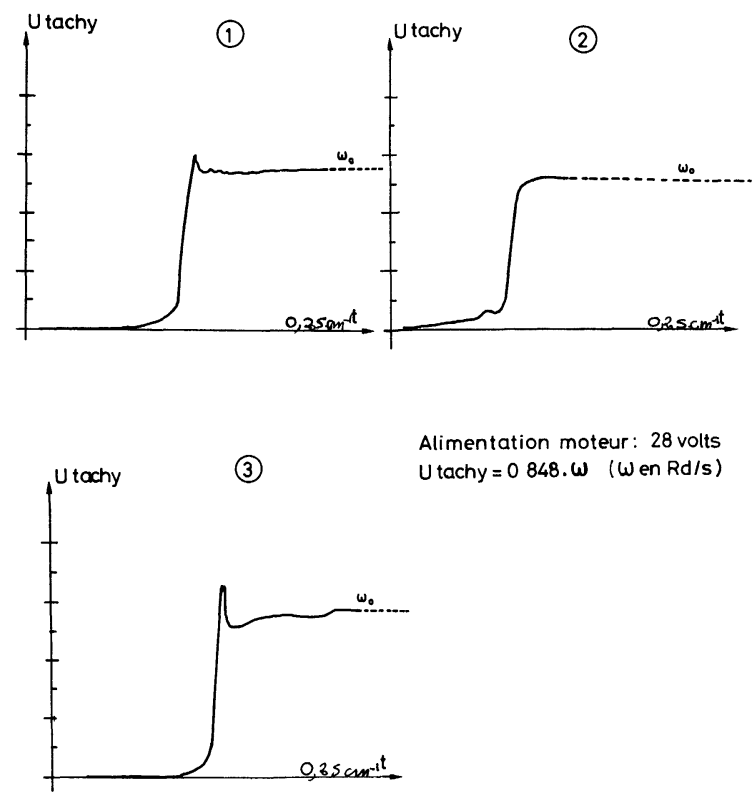

Fig. 5. - Résultats expérimentaux (ex.).

[Experimental data.] 
immobilisé sur le fond pendant une durée supérieure à 24 heures. Dès que la rupture sédiment-métal est faite, le comportement devient identique à celui du frottement étudié ici.

Le dimensionnement du moteur doit, en conséquence être établi à partir de la relation ci-dessous pour ce qui concerne le courant maximal supportable par le moteur :

$$
I=\frac{\sum R S_{n} \tau_{n}}{K}
$$

Le courant en régime permanent $(\partial \omega / \partial t=0)$ vaut, par contre ;

$$
I=\frac{1}{K} \cdot R \cdot F \cdot f(v)
$$

Cette équation définit en fait la valeur du couple en fonction de la force d'avance et de la vitesse. Elle permettra surtout de prévoir la quantité d'énergie à emmagasiner dans les batteries d'accumulateurs en fonction du temps de travail.

La fém des batteries pourra, pour partie, dépendre des conditions de montage. En effet, un prototype ayant deux vis de chaque côté pourrait avoir des motoréducteurs deux à deux et en traînant chacun une vis developpant le même couple de chaque côté dans le cas d'une utilisation rectilique ou bien permettre une régulation en vitesse pour permettre un changement de direction du véhicule car il semble peu probable que ces engins soient équipés de systèmes à pas variables.

\section{Conclusion.}

Le comportement dynamique d'un mobile en appui sur une argile entraîne des pertes énergétiques dues au frottement $f$ qui évolue suivant une loi de la forme $a+b c+c v^{2}$. Ce facteur intervient sur le couple résistant si le vecteur est mû par vis d'archimède et l'équation générale de la vitesse angulaire (ou théorique) en fonction du temps est la suivante :

$$
A=B \frac{\partial^{2} \omega}{\partial t^{2}}+C \frac{\partial \omega}{\partial t}+D \omega+E \omega \frac{\partial \omega}{\partial t}+F^{\prime} \omega^{2}
$$

Des essais confirment cette loi qui peut être simulée par la fonction ci-dessous :

$$
\omega=\omega_{0}\left[1-\exp -\left(\frac{t}{\tau}\right)^{n}\right] .
$$

L'exposant $n$ et la connaissance de $\tau$ calculée pour la fonction théorique et pour les relevés expérimentaux constituent alors des éléments de comparaison.

$\tau$ est plus élevée dans le cas des valeurs expérimentales. La présence d'argile liée au cylindre est une des causes de ce phénomène, (augmentation du frottement et du moment d'inertie global).

L'évolution enregistrée du courant est identique à celle des prévisions déductibles de la relation (14). Son temps de montée est beaucoup plus court que celui de la vitesse angulaire.

Le moteur doit également être apte à vaincre un couple résistant beaucoup plus important que le couple en régime nominal et ceci dans le cas où l'adhérence argile-métal est maximale (égale à la cohésion du sédiment).

\section{Bibliographie}

[1] Aste, J. P., Contribution à l'étude de la locomotion tous terrains sur chenille. Thèse 3e Cycle (1957). Grenoble.

[2] Auriault, J. L., Etude des milieux pulvérulants non chargés dans l'hypothèse de la plasticité parfaite application aux problèmes d'une roue rigide reposant sur du sable. Thèse 3e Cycle (1965), Grenoble.

[3] BESCOND, B., Résistance à l'avancement $R$, opposée par le sol au mouvement d'un véhicule. Thèse 3e Cycle (1970) Grenoble.

[4] BoIsson, Y., Etude de l'adhérence de sédiments argileux à des parois métalliques. Application à la traficabilité sous-marine par vis d'archimède. Thèse $3 \mathrm{e}$ Cycle (1981). Toulouse III.

[5] BouCHENI, M., Etude de la locomotion tous terrainsévolution de roues rigides en milieu pulvérulant à deux et trois dimensions. Thèse 3e Cycle (1964) Grenoble.

[6] BoucheT, J. M., Les véhicules sous-marins au service de la reconnaissance des fonds. J. Rech. Océan. III, nos 2 et 3 (1983).
[7] CoLe, B. N., Engineering into amphibious screw traction. Proceedings of the institution of mecanical. Enginers 175, $\mathrm{n}^{\mathrm{0}} 19$ (1961).

[8] Glasby, G. P., Marine Manganese deposits (Oceanography series. Elsevier. Amsterdam) (1977).

[9] Gibson, D. L. and ANDERson, V. C., Sea floor mechanics and trafficability measurements with the tracked vehicule " $R U M$ » (Deeps sea sediments-Marine Science t2-Plenum Press. New York) (1974).

[10] Longuemard J. P., Plaire, T., Valls A., Locomotion de véhicule sous-marins par vis d'Archimède en appui sur des argiles (étude du rendement). Bilans et perspectives en génie civil (mécanique des sols appliquée). Nancy (1983).

[11] Longuemard, J. P., Monaco, A., Propriétés géotechniques et minéralogiques d'une carotte des fonds à nodule (Pacifique Nord). Application à l'utilisation d'un site côtier de simulation. Océanologica Acta 1, no 4 (1978). 
[12] Moreau, J. P. Automatique et robotique en recherche océanographique. Engins inhabités pour travaux sous-marins. J. Rech. Océan. III nos 2 et 3 (1983).

[13] OBERTI, A., Etude de la locomotion tous terrains. Evolution de roues rigides en milieu pulvérulants. Thèse 3e Cycle (1963) Grenoble.
[14] SChwartz, S., Etude du comportement d'engins en appui et mobiles sur un fond sédimenteux. Application au ramassage des nodules. Rapport de stage (1972), Ecole Centrale de Paris.

[15] Sterenberg, J., Etude de la locomotion tous terrains, étude théorique à deux dimensions des états statiques d'une roue rigide en milieu pulvérulant. (1963) Grenoble. 